\title{
Förderung des wissenschaftlichen Nachwuchses
}

\section{J. Klewer}

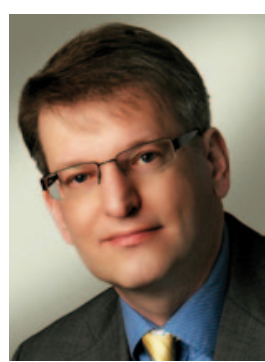

J. Klewer

Dieses Jahr werden Ihnen innerhalb von 4 Wochen 2 Ausgaben von HeilberufeSCIENCE zur Verfügung gestellt: im April das HeilberufeSCIENCE-Supplement anlässlich des 5. HeilberufeSCIENCE-Symposiums im Rahmen des Interprofessionellen Gesundheitskongresses in Dresden und nun die vorliegende aktuelle Ausgabe 02/2015.

Zahlreiche Gespräche rund um das 5. HeilberufeSCIENCESymposium sowie die „poster session“ zeigten, dass v. a. für den wissenschaftlichen Nachwuchs zu wenig Möglichkeiten existieren, eigene Ergebnisse aus kleineren Projekten wissenschaftlich zu publizieren. Nachdem ich vor 5 Jahren erstmalig an dieser Stelle auf die Möglichkeit der Publikation einer wissenschaftlichen Kurzmitteilung in HeilberufeSCIENCE hingewiesen habe, möchte ich nun erneut die Gelegenheit nutzen, auf diese Möglichkeit aufmerksam zu machen. Dank des breiten Themenspektrums von HeilberufeSCIENCE können Nachwuchswissenschaftler schnell erste Erfahrungen hinsichtlich der Publikation von Studienergebnissen sammeln, indem sie beispielsweise auch die relevanten Ergebnisse der eigenen Abschlussarbeiten pub-

\section{J. Klewer $(\triangle)$}

Zwickau, Deutschland

E-Mail: heilberufescience@springer.com lizieren. Somit befördert diese Publikationsform auch den Theorie-Praxis-Transfer.

In der vorliegenden Ausgabe von HeilberufeSCIENCE präsentieren wir Ihnen 3 umfangreiche Originalarbeiten und eine wissenschaftliche Kurzmitteilung. Hermann et al. stellen in ihrer Originalarbeit die Ergebnisse einer Querschnittstudie zur allgemeinen Selbstwirksamkeit von dualen Pflegestudierenden vor. Die Marktmechanismen bei der Preisgestaltung von Heimentgelten für Pflegeheime diskutieren Schaal et al. und gehen zusätzlich mithilfe der Auswertung von 542 Transparenzberichten stationärer Pflegeeinrichtungen auf die Versorgungsqualität ein. Schulc et al. publizieren die Ergebnisse einer Qualitätsüberprüfung des Fragebogens zur subjektiven Einschätzung der funktionalen Gesundheit durch Senioren (F-FG). Die wissenschaftliche Kurzmitteilung von Adner beinhaltet die Ergebnisse einer exemplarischen Untersuchung zur Umsetzung der „Standardebene 1“ des Expertenstandards „Ernährungsmanagement in der Pflege" mit dem Ziel, daraus Maßnahmen zur Optimierung der Struktur-, Prozess- und Ergebnisqualität im Ernährungsmanagement der untersuchten Einrichtung ableiten zu können.

An dieser Stelle möchte ich Sie wieder einladen, die 4 Beiträge dieser Ausgabe von HeilberufeSCIENCE interessiert und kritisch zu lesen - und natürlich würde es mich freuen, wenn ich Sie in der August-Ausgabe von HeilberufeSCIENCE im Sommer wieder an dieser Stelle begrüßen darf.

Ihr

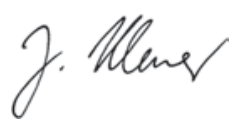

J. Klewer 